\title{
Paediatric crossword puzzle - 29 (Answers)
}

Manouri P Senanayake ${ }^{1}$, Arjuna Atapattu ${ }^{2}$

Sri Lanka Journal of Child Health, 2016; 45(1): 57

DOI: http://dx.doi.org/10.4038/sljch.v45i1.8092

\begin{tabular}{|l|l|l|l|l|l|l|l|l|l|l|l|l|l|}
\hline & & ${ }^{1} \mathrm{I}$ & $\mathrm{N}$ & ${ }^{2} \mathrm{~A}$ & $\mathrm{C}$ & ${ }^{3} \mathrm{C}$ & $\mathrm{U}$ & ${ }^{4} \mathrm{R}$ & $\mathrm{A}$ & $\mathrm{C}$ & $\mathrm{Y}$ & & \\
\hline $\mathrm{P} \mathrm{T}$ & ${ }^{6} \mathrm{~B}$ & & & $\mathrm{R}$ & & $\mathrm{O}$ & & $\mathrm{E}$ & & & & & \\
\hline & $\mathrm{A}$ & & & $\mathrm{M}$ & $\mathrm{E}$ & $\mathrm{N}$ & $\mathrm{S}$ & $\mathrm{T}$ & $\mathrm{R}$ & $\mathrm{U}$ & $\mathrm{A}$ & $\mathrm{L}$ & \\
\hline $\mathrm{I}$ & $\mathrm{L}$ & $\mathrm{E}$ & $\mathrm{U}$ & $\mathrm{S}$ & & $\mathrm{N}$ & & $\mathrm{T}$ & & & & & \\
\hline $\mathrm{N}$ & & & & & & & ${ }^{3} \mathrm{~F}$ & & & & & ${ }^{10} \mathrm{~B}$ & \\
\hline $\mathrm{F}$ & & ${ }^{11} \mathrm{~F}$ & & & ${ }^{12} \mathrm{~S}$ & $\mathrm{E}$ & $\mathrm{L}$ & $\mathrm{F}$ & & ${ }^{13} \mathrm{G}$ & & $\mathrm{E}$ & \\
\hline${ }^{14} \mathrm{~L}$ & $\mathrm{O}$ & $\mathrm{O}$ & $\mathrm{P}$ & & $\mathrm{I}$ & & $\mathrm{E}$ & & & $\mathrm{O}$ & & $\mathrm{E}$ & \\
\hline $\mathrm{I}$ & & $\mathrm{L}$ & & & $\mathrm{X}$ & & ${ }^{15} \mathrm{C}$ & $\mathrm{A}$ & ${ }^{16} \mathrm{R}$ & $\mathrm{R}$ & ${ }^{17} \mathrm{O}$ & $\mathrm{T}$ & \\
\hline $\mathrm{X}$ & & $\mathrm{A}$ & & & & & $\mathrm{A}$ & & $\mathrm{I}$ & & $\mathrm{P}$ & & \\
\hline $\mathrm{I}$ & & $\mathrm{T}$ & & ${ }^{18} \mathrm{~S}$ & $\mathrm{E}$ & $\mathrm{M}$ & $\mathrm{I}$ & & $\mathrm{C}$ & & $\mathrm{I}$ & & \\
\hline $\mathrm{M}$ & & $\mathrm{E}$ & & & & & $\mathrm{N}$ & & $\mathrm{E}$ & & ${ }^{19} \mathrm{~A}$ & $\mathrm{D}$ & ${ }^{20} \mathrm{C}$ \\
\hline $\mathrm{A}$ & & & ${ }^{21} \mathrm{~W}$ & $\mathrm{P}$ & $\mathrm{W}$ & & $\mathrm{I}$ & & & & $\mathrm{T}$ & & $\mathrm{R}$ \\
\hline${ }^{22} \mathrm{~B}$ & $\mathrm{~L}$ & $\mathrm{U}$ & $\mathrm{E}$ & & & & ${ }^{23} \mathrm{D}$ & $\mathrm{E}$ & $\mathrm{A}$ & $\mathrm{N}$ & $\mathrm{E}$ & $\mathrm{R}$ & $\mathrm{Y}$ \\
\hline & & & ${ }^{24} \mathrm{~T}$ & $\mathrm{~A}$ & $\mathrm{~S}$ & $\mathrm{~T}$ & $\mathrm{E}$ & & & & & & \\
\hline
\end{tabular}

${ }^{1}$ Senior Professor in Paediatrics, ${ }^{2}$ Research Assistant in Paediatrics, University of Colombo 